\title{
EXISTENCE THEORY FOR QUADRATIC PERTURBATIONS OF ABSTRACT MEASURE INTEGRO-DIFFERENTIAL EQUATIONS
}

\author{
BAPURAO C. DHAGE
}

Abstract. In this paper, an existence theorem for quadratic perturbations of a nonlinear abstract measure integro-differential equation is proved via a nonlinear alternative of Leray-Schauder type. An existence result is also proved for the extremal solutions for Carathéodory as well as discontinuous cases of the nonlinearities involved in the equations.

Mathematics subject classification (2000): 34G99.

Keywords and phrases: measure integro-differential equation, existence theorem, extremal solutions.

\section{REFERENCES}

[1] B. C. Dhage, On $\alpha$-condensing mappings in Banach algebras, Math. Student, 63 (1994), 146-152.

[2] B. C. DhaGe, On abstract measure integro-differential equations, J. Math. Phy. Sci., 20 (1986), $367-$ 380.

[3] B. C. DHAGE, On system of abstract measure integro-differential inequalities and applications, Bull. Inst. Math. Acad. Sinica, 18 (1989), 65-75.

[4] B. C. Dhage, Periodic boundary value problems of first order Carathéodory and discontinuous differential equations, Nonlinear Funct. Anal. Appl., 13, 2 (2008), 323-352.

[5] B. C. Dhage, D. N. Chate and S. K. Ntouyas, Abstract measure differential equations, Dynamic Systems \& Appl., 13 (2004), 105-108.

[6] B. C. Dhage And S. S. Bellale, Abstract measure integro-differential equations, Global Jour. Math. Anal., 1, 1-2 (2007), 91-108.

[7] B. C. Dhage And D. O'Regan, A fixed point theorem in Banach algebras with applications to nonlinear integral equation, Functional Diff. Equations, 7, 3-4 (2000), 259-267.

[8] A. Granas and J. Dugundu, Fixed Point Theory, Springer Verlag, 2003.

[9] S. HeKkILÄ And V. LaKshmikantham, Monotone Iterative Technique for Discontinuous Nonlinear Differential Equations, Marcel Dekker Inc., New York, 1994.

[10] W. Rudin, Real and Complex Analysis, McGraw-Hill Inc., New York, 1966.

[11] R. R. Sharma, An abstract measure differential equation, Proc. Amer. Math. Soc., 32 (1972), $503-$ 510.

[12] R. R. Sharma, A measure differential inequality with applications, Proc. Amer. Math. Soc., 48 (1975), 87-97.

[13] G. R. Shendge And S. R. Joshi, Abstract measure differential inequalities and applications, Acta Math. Hung., 41 (1983), 53-54. 